\title{
Gastric Ulcers; Causes, Symptoms, Treatment, and Prevention
}

\author{
Kelichi Ndidi \\ Faculty of Health Sciences, National Open University of Nigeria
}

\begin{abstract}
The study discusses the cause, symptom, medication, and prevention of peptic ulcers. The discussion is the main cause of ulcer disease at this time is believed to be due to stomach infection by bacteria we called "Helicobacter pyloricus (H. pylori). the main clinical feature of peptic ulcers is chronic, intermittent epigastric pain which typically subsides after ingestion of food or antacids Some complain of burning in the upper stomach or painful hunger one to three hours after eating or at night when the stomach is empty. Smoking is also an important cause of ulcer formation and failure of ulcer treatment. Treatment of peptic ulcers useds special selection, dual therapy with antibiotics, therapeutic regimens, operation measures.
\end{abstract}

Keywords: Gastric Ulcers, Symptoms, Treatment

\section{Introduction}

The stomach as a food reservoir/barn functions to receive food/drink, shiver, mix and empty food into the duodenum. The stomach, which is always in contact with all types of food, drink, and medicine will experience chronic irritation. The stomach is protected against irritant factors by the mucus layer/mucus berier, epithelium, but several irritant factors such as food and beverages and non-steroidal anti-inflammatory drugs (NSAIDs), alcohol and bile which can cause mucus layer effects and reverse H+ion diffusion, causing gastritis. Acute/chronic and gastric ulcer. (Wallace., 2008).

The discovery of the HP germ as a cause of gastritis and peptic ulcer, it is currently considered that HP is the main cause of gastric ulcers, in addition to NSAIDs, and the rare causes are Zollinger Ellison Syndrome and duodenal Crohn's disease. In this paper, we will discuss peptic ulcers or commonly referred to as gastric ulcers, starting from understanding to managing the disease both therapeutically and non-therapeutically.

\section{Definition of gastric Ulcers}

According Sibilia et al. (2003), Peptic ulcer/benign gastric ulcer is a round or semiround/oval image, size $>5 \mathrm{~mm}$ into the sub mucosa of the gastric mucosa due to a break in the continuity / integrity of the gastric mucosa. Gastric ulcers are open sores with edematous margins accompanied by induration with the base of the ulcer covered with debris. (Nigam \& Paarakh, 2011).

According to Grassi et al. (2011), Peptic ulcer is a disease caused by disorders of the upper gastrointestinal tract caused by excessive secretion of acid and pepsin by the gastric mucosa. By definition, peptic ulcers can be found in any part of the digestive tract affected by gastric juice, namely the esophagus, stomach, duodenum and after gastroenterostomy, as well as the jejenum. Gastric ulcers, occur when the stomach wall is damaged due to the damaged mucus covering it. The enzymes produced in the mucus eat small parts in the surface layer of the stomach. This disease caused by viral infection causes the stomach wall to become perforated so that the contents fall into the abdominal cavity. According to Ravisankar et al. (2016) A peptic ulcer is an opening in the lining of the duodenum, stomach or esophagus. An

Copyright (C) 2020, Journal Wetenskap Health, Under the license CC BY-SA 4.0 
ulcer that is in the stomach is called a stomach ulcer, an ulcer that is in the duodenum is called a duodenal ulcer and an ulcer that is in the esophagus is called an esophageal ulcer.

Therefore, Ulcers occur when the lining of these organs is corroded by acidic digestive juices secreted by gastric cells. Peptic ulcer disease is common.

\section{Causes of gastric ulcers}

Excess acid is believed to be the main cause of ulcer disease. Therefore, the emphasis of treatment is on neutralizing and inhibiting gastric acid secretion. Meanwhile, according to Franceschi et al. (2014), acid is still considered significant in the formation of ulcers, the main cause of ulcer disease today which is believed to be due to stomach infection by a bacterium called "Helicobacter pyloricus (H. pylori). Another major cause of ulcers is the use of chronic anti-inflammatory drugs, often referred to as NSAIDs (anti-inflammatory drugs), including aspirin. Smoking is also an important cause of ulcer formation and failure of ulcer treatment.

Pylori is a very common bacteria. These bacteria infect more than one billion people worldwide. It is estimated that half of the United States population over the age of 60 has been infected with H. pylori (Parsonnet et al. 1996)

Infection usually lasts for years, causing ulcers in $10 \%$ to $15 \%$ of those infected with $\mathrm{H}$. pylori found in more than $80 \%$ of patients with gastric and duodenal ulcers. Meanwhile, the mechanism by which $\mathrm{H}$. pylori causes ulcers is not well understood. Elimination of these bacteria with antibiotics has clearly been shown to prevent recurrence of ulcers. According Brewer et al. (2010), NSAIDs are drugs for arthritis and painful inflammatory conditions in the body. NSAIDs cause changes in the quality of gastric mucus which can facilitate the degradation of mucus by pepsin. Aspirin, ibuprofen (Motrin), naproxen (Naprosyn), and etodolac (Lodine) are examples of this class of drugs. Prostagladins are important substances in helping to fight damage to the intestinal lining. Corrosive acids. Excessive amounts of prostaglandins play an important role in gastric mucosal defense. NSAIDs cause ulcers by disrupting prostaglandins in the stomach.

\section{Symptoms and Clinical Features of Gastric Ulcers}

The main clinical feature of peptic ulcers is chronic, intermittent epigastric pain which typically subsides after ingestion of food or antacids (Sipponen \& Maaroos., 2015). Some complain of burning in the upper stomach or painful hunger one to three hours after eating or at night when the stomach is empty. This pain is often described as slicing, burning or feeling bad. This pain-eating-disappearing pattern may be atypical in gastric ulcers. Even in some people with stomach ulcers, food can make the pain worse. Usually, people with stomach ulcers will experience weight loss, while people with duodenal ulcers usually have a fixed weight.

\section{Treatment and Prevention of Gastric Ulcers}

\section{Special selection}

Patients with positive HP who received eradication were divided into three groups including (1) Highly recommended: duodenal ulcers, gastric ulcers, post-resection of early gastric cancer, MALT lymphoma; (2) Recommended: ulcer-type dyspepsia. Severe active chronic gastritis (PA picture), NSAID symptoms, severe erosive gastritis, hypertrophic gastritis; (3) Not recommended: asymptomatic patients (HP Indonesia Study Group, KSHPI. Currently a consensus has been agreed upon, among others: NHI / national institute of Health 
consensus development (USA) American Digestive Health Foudation, European Maastricht consensus, Asia pacific consensus conference.

Consequences: HP in peptic ulcers is recommended to be eradicated, regardless of whether or not to eradicate, regardless of whether the first episode or not, the severity of the errors, the presence of aggravating factors such as NSAIDs or the duration of ulcer remission. Ulcers with positive HP (serology validated UBT funds), it is recommended to eradicate, MALT Lymphoma will experience more than half of it if HP eradication. Eradication of HP in non-ulcer dyspepsia to prevent gastric malignancy or in GERD patients where drug use must be given a long time is controversial.

\section{Dual Therapy with Antibiotics}

If a dual therapy between PPI / ARH2 and one antibiotic is to be given, it is not recommended because: the eradication effect is minimal, less than $80 \%$ and quickly causes germ resistance.

\section{Therapeutic Regime}

Historically, the first eradication therapy regimens used were: Bismuth, Metronidazole, Tetracycline. The triple therapy regimen (PPI 2x1, Amoxicilin 2x1000, Claritomycin 2x500, Metronidazole $3 \times 500$, Tetracycline $4 \times 500$ ) is widely used today. Duration of HP eridication treatment 1 week (esomesoprazole), 5 days rabeprazole. There is a suggestion for 2 weeks of eradication treatment, for ulcer healing, you can continue giving PPI for another 3-4 weeks. eradication success should be above $90 \%$ side effects of triple therapy 20-30\%. Failure of eradication treatment is usually due to side effects and compliance and germ resistance. Infection within 6 months of forced erasication is usually a recruitment for other bacterial infections. Purpose of HP education: (1). Reducing complaints / symptoms, (2) Healing ulcers and (3) Prevent recurrence (4\% versus 59\% TL, 6\% versus 67\% / BP). Eradication can prevent recurrence of ulcers and also prevent bleeding and malignancy. Quadriple therapy. If triple therapy fails, then it is recommended to provide a quadruple therapy regimen, namely: $2 \mathrm{x}$ daily PPI, Bismuth subsalicylate $4 \times 2$ tab, MNZ 4x250, Tetracycline $4 \times 500$, if bismuth is not available, replaced with triple therapy.

\section{Operating Measures}

Operation of surgery when the frequency decreases due to the success of medical therapy and endocopin therapy. Refractory ulcers are now rare. The surgical procedure performed in gastric ulcer disease determines the inclusion of duodenal ulcers: (1) Anastomic ulcer was performed anteroctomy (including ulcer) and Bilroth 1 gastroduodenostomy anastomosis, if included BP was performed vagotomy. The high incidence of recurrent forced ulcer surgery means that this procedure is less desirable, (2) Gastric ulcer near EG junction surgery is performed more radical/sub total gastrectomy with Roux-en-Y/esophagogastro jejunostomy (Csendo procedure). If the patient is in poor condition, the location of the proximal ulcer is performed with a Madlener kelling procedure including anterectomy, intraoperative ulcer biopsy and vagotomy, ulcer recurrence is $30 \%$.

\section{Conclusion}

From the writing of this paper, it can be concluded that gastric ulcers occur when the stomach wall is damaged due to damaged mucus covering it. The enzymes produced in the mucus eat small parts in the surface layer of the stomach. This disease caused by viral infection causes the stomach wall to become perforated so that the contents fall into the abdominal cavity. The main cause of stomach ulcers is believed to be due to a stomach

Copyright (C) 2020, Journal Wetenskap Health, Under the license CC BY-SA 4.0 
infection by a bacteria called Helicobacter pyloricus (H. pylori). Another major cause of ulcers is the use of chronic anti-inflammatory drugs, often referred to as NSAIDs (antiinflammatory drugs), including aspirin. Smoking is also an important cause of ulcer formation and failure of ulcer treatment. Treatment that can be done to treat stomach ulcers is with antacid drugs that can neutralize stomach acid, histamine $\mathrm{H} 2$ blocker antagonists. Histamine antagonists ( $\mathrm{H} 2$ blockers) are drugs designed to block the action of histamine on stomach cells thereby reducing acid output, for example cimetidine, ranitidine and famotidine. It is hoped that we can protect our stomachs from food and drinks that enter the body from being infected by the Helicobacter pylori bacteria and also not underestimating stress. Because when stress increases, stomach acid production causes the $\mathrm{pH}$ in the stomach to become acidic so that it can damage the stomach lining. And it is also advisable to stop smoking because smoking is the cause of failure in the treatment of stomach ulcers. Reference

\section{Refferences}

Brewer, A. R., McCarberg, B., \& Argoff, C. E. (2010). Update on the use of topical NSAIDs for the treatment of soft tissue and musculoskeletal pain: a review of recent data and current treatment options. The Physician and sportsmedicine, 38(2), 62-70.

Cone, R. A. (2009). Barrier properties of mucus. Advanced drug delivery reviews, 61(2), 7585.

Franceschi, F., Zuccalà, G., Roccarina, D., \& Gasbarrini, A. (2014). Clinical effects of Helicobacter pylori outside the stomach. Nature Reviews Gastroenterology \& Hepatology, 11(4), 234-242.

Grassi, M., Petraccia, L., Mennuni, G., Fontana, M., Scarno, A., Sabetta, S., \& Fraioli, A. (2011). Changes, functional disorders, and diseases in the gastrointestinal tract of elderly. Nutricion hospitalaria, 26(4), 659-668.

Nigam, V., \& Paarakh, P. M. (2011). Anti-ulcer effect of Chenopodium album Linn. against gastric ulcers in rats. International Journal of Pharmaceutical Sciences and Drug Research, 3(4), 319-322.

Parsonnet, J., Harris, R. A., Hack, H. M., \& Owens, D. K. (1996). Modelling costeffectiveness of Helicobacter pylori screening to prevent gastric cancer: a mandate for clinical trials. The Lancet, 348(9021), 150-154.

Ravisankar, P., Koushik, O., Reddy, A., KumarU, A. P., \& Pragna, P. (2016). A Detailed Analysis on Acidity and Ulcers in Esophagus, Gastric and Duodenal Ulcers and Management. IOSR Journal of Dental and Medical Sciences (IOSR-JDMS), 15(1), 94-114.

Sipponen, P., \& Maaroos, H. I. (2015). Chronic gastritis. Scandinavian journal of gastroenterology, 50(6), 657-667.

Sibilia, V., Rindi, G., Pagani, F., Rapetti, D., Locatelli, V., Torsello, A., ... \& Netti, C. (2003). Ghrelin protects against ethanol-induced gastric ulcers in rats: studies on the mechanisms of action. Endocrinology, 144(1), 353-359..

Wallace, J. L. (2008). Prostaglandins, NSAIDs, and gastric mucosal protection: why doesn't the stomach digest itself?. Physiological reviews, 88(4), 1547-1565. 